\title{
HIV-1 fusion peptide targets the TCR and inhibits antigen-specific $T$ cell activation
}

\author{
Francisco J. Quintana, ${ }^{1}$ Doron Gerber, ${ }^{2}$ Sally C. Kent, ${ }^{3}$ Irun R. Cohen, ${ }^{1}$ and Yechiel Shai ${ }^{2}$
}

${ }^{1}$ Department of Immunology and 2Department of Biological Chemistry, Weizmann Institute of Science, Rehovot, Israel.

${ }^{3}$ Center for Neurologic Diseases, Harvard Institute of Medicine, Boston, Massachusetts, USA.

\begin{abstract}
The fusion peptide (FP) in the N terminus of the HIV envelope glycoprotein, gp41, functions together with other gp41 domains to fuse the virion with the host cell membrane. We now report that FP colocalizes with CD4 and TCR molecules, coprecipitates with the TCR, and inhibits antigen-specific $T$ cell proliferation and proinflammatory cytokine secretion in vitro. These effects are specific: $T$ cell activation by PMA/ionomycin or mitogenic antibodies is not affected by FPs, and FPs do not interfere with antigen-presenting cell function. In vivo, FPs inhibit the activation of arthritogenic $T$ cells in the autoimmune disease model of adjuvant arthritis and reduce the disease-associated IFN- $\gamma$ response. Hence, FPs might play 2 roles in HIV infection: mediating membrane fusion while downregulating $\mathrm{T}$ cell responses to itself that could block infection. Disassociated from HIV, however, the FP molecule provides a novel reagent for downregulating undesirable immune responses, exemplified here by adjuvant arthritis.
\end{abstract}

\section{Introduction}

HIV infection confounds the immune response (1). Untreated HIV infection usually leads to AIDS, a state of general immunosuppression, and susceptibility to otherwise innocuous opportunistic infections $(2,3)$. However, to establish a successful infection and replicate, the virus has to evade immune control, a task that HIV accomplishes by using a broad array of mechanisms, recently reviewed (4). Of particular interest is the inhibition of the $\mathrm{CD}^{+} \mathrm{T}$ cell activity directed to $\operatorname{HIV}$ itself $(5,6)$; anti-HIV $\mathrm{CD}^{+}{ }^{+} \mathrm{T}$ cells are required to establish a $\mathrm{CD}^{+} \mathrm{T}$ cell response capable of controlling the virus (7). Elucidation of the mechanisms used by HIV to downregulate $\mathrm{CD}^{+} \mathrm{T}$ cell activity should improve our understanding of the development of AIDS following HIV infection.

HIV infection of target cells requires fusion of the viral membrane with the cellular membrane; this process is catalyzed by the product of the env gene, the HIV envelope glycoprotein gp160. Mature gp160 is composed of 2 noncovalently associated subunits - gp120 and gp41 (8). Following the interaction of gp120 with membrane receptors on the target cell, the gp41 subunit plays a critical role in virus entry into the target cell. Several functional domains have been identified in gp41 (Figure 1). The $\mathrm{N}$ terminal hydrophobic fusion domain, the fusion peptide (FP), is thought

Nonstandard abbreviations used: AA, adjuvant arthritis; AMP, amphipathic peptide; AMP-Rho, AMP conjugated to rhodamine; $\mathrm{CP}$, core peptide corresponding to the TCR- $\alpha$ transmembrane domain; DCC, $N, N$-dicyclohexylcarbodiimide; DMF, dimethylformamide; DTH, delayed-type hypersensitivity; F9L, mutant transmembrane domain 4 from crylAc $\delta$-endotoxin, in which Phe is mutated to Leu; FP, fusion peptide; FP-NBD, fusion peptide conjugated to 4-chloro-7-nitrobenz-2-oxa-1,3diazole fluoride; FP-Rho, FP conjugated to rhodamine; FRET, fluorescence energy transfer; gp160, HIV envelope glycoprotein gp160; HSP65, 65-kDa heat shock protein; IFA, incomplete Freund's adjuvant; ISU, immunosuppressive domain of HIV gp41; MBP85-99, peptide corresponding to the 85-99 region of human myelin basic protein; Mt, Mycobacterium tuberculosis; Mt176-190, peptide corresponding to the 176-190 region of the HSP65 protein of M. tuberculosis; NBD, 4-chloro-7-nitrobenz-2-oxa-1,3diazole fluoride; PPD, tuberculin-purified protein derivative; rhodamine-SE, 5-carboxytetramethylrhodamine succinimidyl ester; TFA, trifluoroacetic acid; V2E, FP with a V-to-E mutation at position 513; V2E-Rho, V2E peptide conjugated to rhodamine. Conflict of interest: The authors have declared that no conflict of interest exists.

Citation for this article: J. Clin. Invest. 115:2149-2158 (2005).

doi:10.1172/JCI23956. to play a central role in membrane fusion (Figure 1). Indeed, a mutant with a single aa substitution, V2E, shows less fusogenic activity than a wild-type FP (9). The first 16 aa of FP insert into the target cell membrane, and the $\mathrm{C} 20$ region inserts into the virus membrane $(10,11)$. The N36 and C34 peptides contain heptad repeats that form a 6 -helix bundle linker (12) that brings the viral and target membranes into close proximity. Fusion can be inhibited by a peptide corresponding to the $\mathrm{C}$ terminal heptad repeat, DP178; this peptide is a potent inhibitor of HIV infection and has recently been approved for use in humans (13).

With regard to the interaction of FP with $\mathrm{T}$ cell membranes, Cladera et al. reported that a synthetic peptide encoding the 16 $\mathrm{N}$ terminal aa of FP shows a heterogeneous distribution on the membrane of the Jurkat $\mathrm{T}$ cell line (14). We have recently reported that the 33-aa FP inserts into microdomains on T cells (15). Moreover, this FP showed a higher affinity toward ordered membrane domains in vitro (15). Assembly of the TCR and the CD4 molecules as well as other key molecules is required for complete $\mathrm{T}$ cell activation $(16,17)$. Therefore, we reasoned that FP, if it inserts itself into membrane domains containing these $\mathrm{T}$ cell complexes, might interfere with $\mathrm{T}$ cell activation.

In this study we analyzed the membrane distribution of FP in $\mathrm{T}$ cells and the effect of FP on $\mathrm{T}$ cell activation. We found that FP colocalizes with the TCR and CD4 molecules and inhibit T cell activation in vitro and in vivo. These results suggest a role for FP in the downregulation of HIV-specific immunity.

\section{Results}

FP colocalizes with CD4 and TCR. We studied the distribution of FP in the membrane of activated rat A2b T cells using FP conjugated to rhodamine (FP-Rho) or to 4-chloro-7-nitrobenz-2-oxa-1,3-diazole fluoride (NBD; FP-NBD). Rather than uniformly labeling the T cell membrane, both FP-Rho (Figure 2A) and FP-NBD (data not shown) showed a heterogeneous membrane distribution. This distribution in membrane domains contrasted with that of a control membrane-active amphipathic peptide conjugated to rhodamine (AMP-Rho), which demonstrated a uniform distribution on the cell membrane (Figure 2A). 


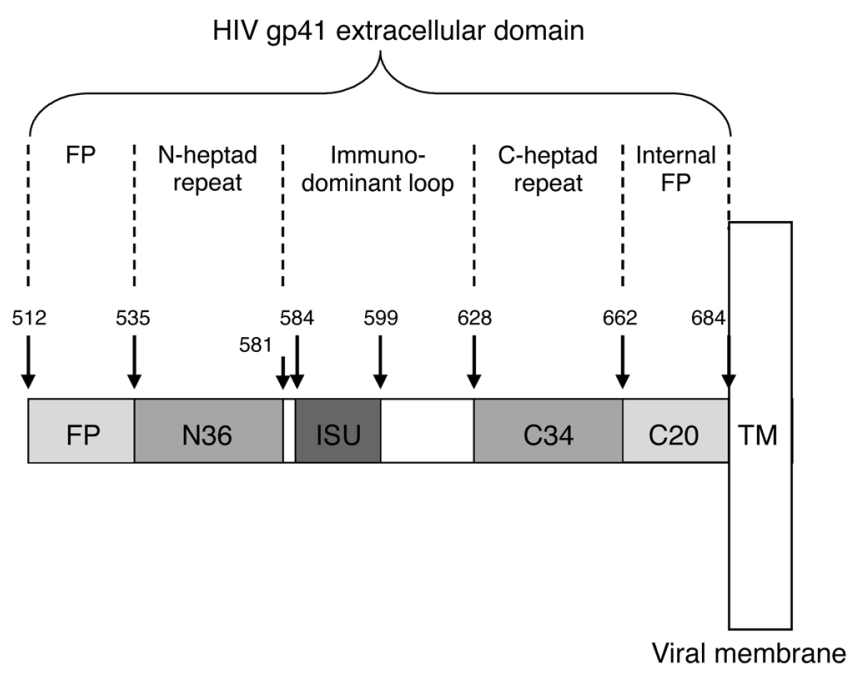

The TCR, CD3, and CD4 molecules, among other components, are found in microdomains in the membranes of activated $\mathrm{CD} 4^{+}$ $\mathrm{T}$ cells (18). Figure 2B shows the localization of the CD4 and TCR molecules in the membrane. Note that the FP conjugates colocalized with the CD4 and TCR molecules (Figure 2B). Both FP-Rho (Figure 2B) and FP-NBD (data not shown) showed the same colocalization; hence, the distribution of the FP with the CD4 and TCR molecules on the $T$ cell membrane did not result from the use of a particular fluorophore. In addition, an FP mutant V2E peptide conjugated to rhodamine (V2E-Rho; Figure 1 and Table 1), showed a similar colocalization with CD4 (data not shown) and the TCR (Figure 2C).

We repeated these procedures with cells that were not treated with fixative, and the results were the same (data not shown). When resting A2b cells were used, colocalization of FPs with CD4 and the TCR molecules was still observed, but there was no cap and much less aggregation of the TCR molecules on the surface of the membrane (data not shown).

We confirmed the colocalization of FP and its V2E mutant with the TCR and CD4 receptors by fluorescence energy transfer (FRET) (Figure 2D). Using a 543-nm laser, we bleached a spot on the cell membrane, thereby reducing the fluorescence of the FP-Rho or V2E-Rho conjugates at that particular spot. A significant increase in the fluorescence of the labeled TCR was observed due to FRET between the labeled, bleached FP (acceptor) and a TCR-specific FITC-conjugated antibody (donor). The average $\mathrm{R}_{0}$ (the characteristic Forester distance at which FRET efficiency is 0.5 ) is under $50 \AA$ for the FITC/Rho pair (19); therefore, the detection of FRET (Figure 2D) indicates that the donor and acceptor molecules must be fewer than $50 \AA$ apart in the membrane. These results suggest that FP binds to the T cell membrane and preferentially colocalizes with the CD4 and TCR molecules.

$F P$ interferes with $T$ cell activation in vitro. Does the colocalization of FP with the CD4 and TCR molecules interfere with $\mathrm{T}$ cell activation? To explore this question, we prepared lymph node cells from Mycobacterium

\section{Table 1}

\section{Figure 1}

HIV-1 gp41 extracellular domain. The extracellular portion of gp41 (aa 512-684) contains a number of functional domains: gp41 becomes active after gp120 (aa 1-511) binds to surface receptors; FP inserts into the membrane of the target cell; $\mathrm{C} 20$ inserts into the membrane of the virion; N36 and C34 form a 6-helix bundle "spring" that brings the membranes into apposition; and ISU is immunosuppressive. TM, gp41 transmembrane domain.

tuberculosis-immunized (Mt-immunized) rats and studied their $\mathrm{T}$ cell responses to the mycobacterial antigens tuberculin-purified protein derivative (PPD) and the peptide corresponding to the 176-190 region of M. Tuberculosis HSP65 (Mt176-190), which contains the 180-188 epitope of the mycobacterial $65-\mathrm{kDa}$ heat shock protein (HSP65). These antigens are known to induce strong proliferative responses and cytokine release from $\mathrm{T}$ cells in the draining lymph node cells of Mt-immunized rats $(20,21)$.

Figures $3 \mathrm{~A}$ and $3 \mathrm{~B}$ show that FP and the V2E mutant peptide inhibited the $\mathrm{T}$ cell proliferative responses to PPD and to Mt176-190 in a dose-dependent manner. Moreover, the inhibitory effect of V2E was lower than that of FP, suggesting that inhibition is sequence specific and that critical molecular interactions were perturbed by the V-to-E substitution in V2E (see Figure 1 and Table 1). The effective $\mathrm{IC}_{50}$ observed for FP was 280 nM. However, some of the peptide self aggregated and only a fraction of FP was available to insert itself into the T cell membrane, as will be discussed later. The FP, and to a lesser extent $\mathrm{V} 2 \mathrm{E}$, also inhibited in a dose-dependent manner the secretion of IFN- $\gamma$ and IL-10 triggered by stimulation with PPD or the Mt176-190 peptide (Figure 3, C-F). The inhibitory effects of the FP and V2E peptides on antigen-triggered proliferation and cytokine release were not due to cell death, since cells incubated with FP, V2E, or the control peptide p277 showed the same survival in culture (data not shown).

We then studied the effects of FP on the activation of human $\mathrm{T}$ cells using a human Th0 cell clone (Ob1A12) reactive with the 85-99 region of human myelin basic protein (22); this epitope is contained in the MBP85-99 peptide used in our studies (Table 1). The activation of the Ob1A12 T cell clone with the MGAR B cell clone fed with the MBP85-99 peptide triggers Ob1A12 proliferation and secretion of both IFN- $\gamma$ and IL-13. Figure 4A shows that FP inhibited the proliferative response of Ob1A12 to MBP85-99 in a dose-dependent manner. No inhibition was detected when the control peptide core peptide mutant $(\mathrm{CP}-2 \mathrm{G})(23,24)$ was used.

Designations and sequences of peptides

$\begin{array}{lc}\text { Designation } & \text { Start } \\ \text { FP } & 512 \\ \text { V2E } & 512 \\ \text { AMP } & - \\ \text { p277 } & 437 \\ \text { Mt176-190 } & 176 \\ \text { F9L } & 125 \\ \text { MBP85-99 } & 85 \\ \text { CP-2G } & -\end{array}$

aa sequence
AVGIGALFLGFLGAAGSTMGARSMTLTVQARQL
AEGIGALFLGFLGAAGSTMGARSMTLTVQARQL
LLKLLKKLLKKLLKL
VLGGGCALLRCIPALDSLTPANED
EESNTFGLQLELTEG
LREEMRIQLNDMNSALTTAIPLFA
ENPVVHFFKNIVTPR

End

535

535

$-$

460

190

148

99

$-$

Protein
gp41
gp41
Synthetic
Human HSP60
Mt HSP65
Cry1Ac
MBP
Rat TCR- $\alpha$

Start and end positions are designated according to the HIV-1 HXB2 strain gp160 sequence. The valine to glutamic acid mutation at position 2 of the FP is marked in bold. 
The FP also inhibited in a dose-dependent manner the secretion of IL-13 and, to a lesser extent, the secretion of IFN- $\gamma$ (Figure 4, B and C). No such inhibitory effects were seen when the control peptide CP-2G was used. The inhibitory effects of the FP on antigen-triggered proliferation and cytokine release of human $T$ cells were not due to cell death: cells incubated with FP or the control peptide CP-2G showed the same degree of survival in culture (data not shown). Thus, FP can also interfere with the activation of human $\mathrm{T}$ cells in vitro.

FP acts on the Tcells and not on the antigen-presenting cells. The inhibitory effects of FP on $\mathrm{T}$ cell activation were further studied using a rat $\mathrm{CD}^{+} \mathrm{T}$ cell clone, $\mathrm{A} 2 \mathrm{~b}$, which proliferates and secretes IFN- $\gamma$ upon stimulation with the Mt176-190 peptide (20, 21). Activation of A2b by Mt176-190 in the presence of FP led to decreased cell proliferation (Figure 5) and IFN- $\gamma$ secretion (data not shown).

To define the cells targeted by FP in the inhibition of T cell activation, we preincubated separately with $\mathrm{FP}$ the $\mathrm{A} 2 \mathrm{~b} \mathrm{~T}$ cells or the APCs before mixing the cells together with the Mt176-190 peptide. Preincubation of the APCs had no effect on A2b proliferation (Figure 5). However, preincubation of the A2b T cells with the FP, and not with the control peptide $\mathrm{p} 277$, led to a significant inhibition of $\mathrm{T}$ cell proliferation (Figure 5). Thus, FP inhibits $\mathrm{T}$ cell activation by directly acting on the T cells rather than on the APCs.

$F P$ does not inhibit $T$ cell activation induced by PMA/ionomycin or antibodies to $C D 3$. To learn whether FP can also inhibit $\mathrm{T}$ cell activation other than that induced by APC presentation of specific antigen, we tested the effect of FP on T cell activation induced by PMA/ ionomycin or a mitogenic monoclonal antibody to CD3. FP did not inhibit the activation of $\mathrm{A} 2 \mathrm{~b} \mathrm{~T}$ cells by either $\mathrm{PMA} /$ ionomycin (Figure 6A) or mitogenic anti-CD3 (Figure 6B). PMA/ionomycin activates the $T$ cell downstream of the membrane, and mitogenic anti-CD3 antibodies activate CD3 signaling regardless of the presence or absence of the TCR (23). These findings suggest that FP interferes with $\mathrm{T}$ cell activation induced by the recognition of the MHC-peptide complex presented by APCs, probably interfering with TCR/CD3 crosstalk.

FP interacts with the TCR. To identify the targets of FP in the T cell membrane, we incubated the T cells with FP-Rho and cross-linked the complexes formed using $N, N$-dicyclohexylcarbodiimide (DCC). DCC is a hydrophobic cross-linker that forms covalent bonds between carboxyl groups and positive charges within the membrane milieu (25). The FP displayed a specific pattern of binding to about 7 unidentified $\mathrm{T}$ cell proteins in 4 separate experiments (Figure 6C, lane 8). In comparison, the mutant transmembrane domain 4 from crylAc $\delta$-endotoxin, in which Phe is mutated to Leu (F9L) conjugated to rhodamine (F9L-Rho), a control transmembrane domain (26), did not bind any $\mathrm{T}$ cell protein in detectable amounts (Figure 6C, lane 10). The monomeric form of the peptides is too small to be detected by the lower range of the gel, which is about $11 \mathrm{kDa}$; however FP also shows high oligomeric forms corresponding to trimer and heptamer (Figure 6C, lane 7). F9L has no oligomeric form larger than the lower limit (Figure $6 \mathrm{C}$, lane 9). Total protein staining of the SDS-PAGE gel with Coomassie brilliant blue confirmed that the observed differences were not due to different concentrations of $\mathrm{T}$ cell proteins. We found no effect on $T$ cell protein expression by the peptides when comparing Coomassie staining of peptides cross-linked with wholecell extract (Figure 6C, lanes 4 and 5) with Coomassie staining of whole-cell extract alone (Figure 6C, lane 6). The amount of fluorescent FP or F9L loaded without cells in Figure 6C was 2 pmoles,

but peptides alone are not visible with Coomassie staining (Figure $6 \mathrm{C}$, lanes 2 and 3 ). However, the fluorescence intensity of the free FP bands (Figure 6C, lane 7) was higher than the total intensity of the FP-labeled targets (Figure 6C, lane 8). Moreover, the sizes of the 2 bands observed for FP in the absence of whole T cell extract confirm aggregation of FP in solution (Figure 6C, lane 7). Thus, we may conclude that the actual concentration of FP available for immunosuppression is much lower than the nominal concentration used in each experimental condition.

Attempts to isolate and identify the targets of FP by mass spectrometry were unsuccessful. Therefore, we analyzed the interaction of FP with $\mathrm{T}$ cell proteins by immunoprecipitation. Based on the FRET between the TCR and FP, we tested whether we could immunoprecipitate the labeled FP-Rho with antibodies against TCR. Antibodies against actin, CD28, HSP60, or MHC class I were used as controls. FP-Rho coprecipitated with the TCR, but

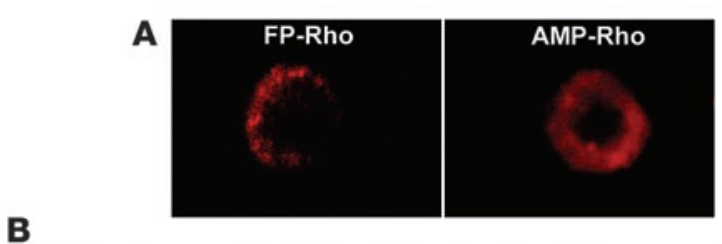

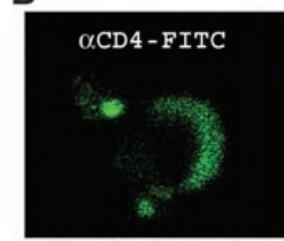

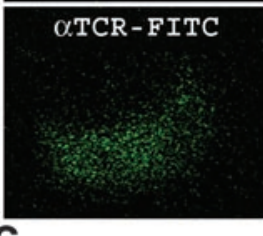

C


Figure 2

FP colocalizes with the CD4 and TCR molecules in the T cell membrane. FP-Rho, V2E-Rho, and AMP-Rho were used to study peptide binding to the membranes of activated $A 2 b T$ cells in combination with FITC-labeled antibodies to CD4 or TCR. The A2b T cells had been activated by incubation with APC and the Mt176-190 peptide. (A) Distribution of FP-Rho and AMP-Rho in activated T cells. (B) Colocalization of FP-Rho with the CD4 and TCR molecules. (C) Colocalization of V2E-Rho with the TCR. (D) FRET between FP-Rho or V2E-Rho and FITC-labeled antibodies to TCR is confirmed by increase of FITC (donor) intensity after bleaching of a spot of rhodamine (acceptor) at $543 \mathrm{~nm}$. Scale bar: $5 \mu \mathrm{m}$. 

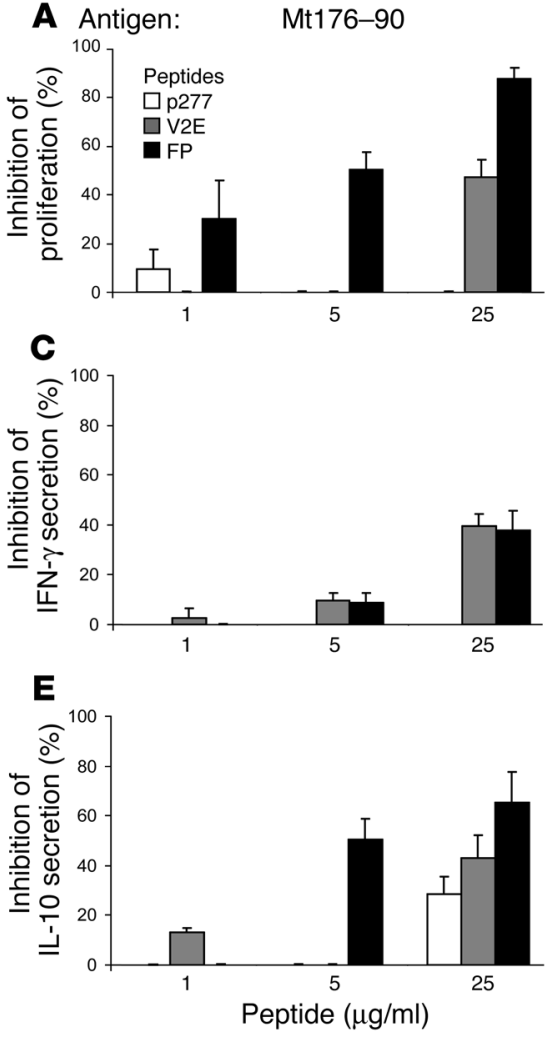
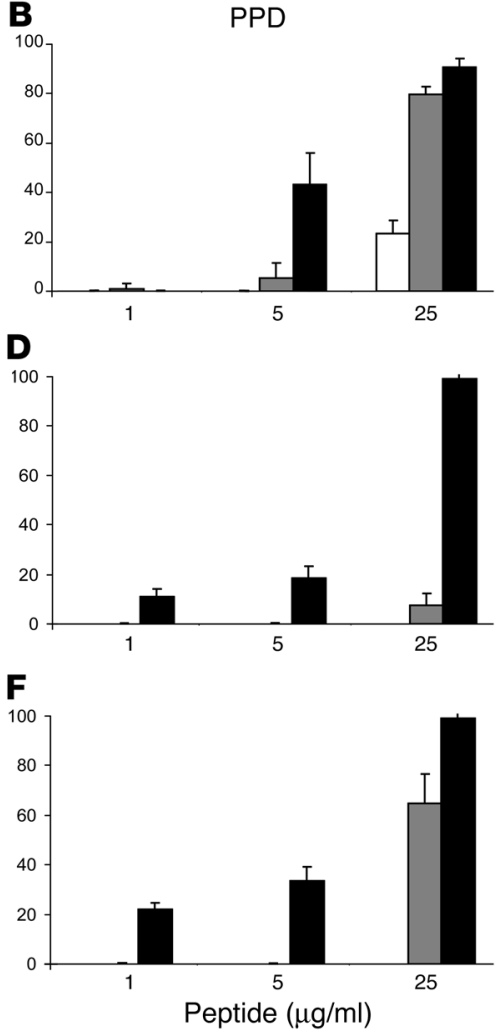

\section{Figure 3}

FP inhibits the T cell response to Mt. Lymph node cells from Mt-immunized rats were activated in vitro with Mt176-190 (A, C, and E) or PPD (B, D, and F) in the presence of FP, V2E, or p277. Proliferation (A and $\mathbf{B}$ ), IFN- $\gamma$ secretion (C and $\mathbf{D}$ ), and IL-10 secretion ( $\mathbf{E}$ and $\mathbf{F}$ ) were assayed. The data are presented as mean inhibitions $\pm \mathrm{SD}$ ( $n=3$ or more). The uninhibited T cell proliferative responses were $12258 \pm 578 \mathrm{cpm}$ and $1488 \pm 103 \mathrm{cpm}$ for PPD and Mt176-190, respectively. The background proliferation in the absence of antigen (8-10 days after a previous activation by incubation with APC and PPD or the Mt176-190 peptide) was $380 \pm 15 \mathrm{cpm}$. IFN- $\gamma$ secretion was $1155 \pm 254$ $\mathrm{pg} / \mathrm{ml}$ and $1289 \pm 310 \mathrm{pg} / \mathrm{ml}$ for cells activated by PPD and Mt176-190, respectively. IL-10 secretion was $364 \pm 69 \mathrm{pg} / \mathrm{ml}$ and $314 \pm 23 \mathrm{pg} / \mathrm{ml}$ for cells activated by PPD and Mt176-190, respectively. not with actin, CD28, HSP60, or MHC class I (Figure 6D). Conversely, the control peptide F9L-Rho did not coimmunoprecipitate with any of these molecules; only insignificant unspecific binding was detected (Figure 6D).

We have previously shown that the $\mathrm{V} 2 \mathrm{E}$ mutant colocalizes with TCR and CD4 molecules on the T cell surface (Figure 2); however, V2E is less effective than FP in inhibiting $\mathrm{T}$ cell activation (Figures 3 and 5). Thus, we analyzed whether a labeled V2E mutant (V2E-Rho) could be immunoprecipitated with the TCR or with MHC I molecule as a control. Figure $6 \mathrm{E}$ shows that the V2E-Rho mutant did not immunoprecipitate with the TCR or MHC I, highlighting the specificity of the FP/TCR interaction involved in the inhibition of $\mathrm{T}$ cell activation by $\mathrm{FP}$.

FP inhibits $T$ cell immunity in vivo. To test the inhibitory effects of FP on the activation of specific T cells in vivo, we studied the effects of FP on adjuvant arthritis (AA). The immunization of Lewis rats with $\mathrm{Mt}$ in oil triggers $\mathrm{AA}$, an experimental autoimmune disease driven by Mt-specific T cells cross-reactive with self antigens $(27,28)$. Mt176-190-specific T cells are detectable upon induction of AA (29); indeed the A2b T cell clone, which reacts with Mt176-190, cross-reacts with cartilage and mediates AA (30). Since FP inhibited the T cell response of primed lymph node cells and of the A2b clone to PPD and Mt176-190 in vitro (Figures 3 and 5), we also investigated the effects of FP on the in vivo activation of the T cells that drive AA.

Figure 7 shows that FP administered with the antigen at the time of AA induction led to a significantly milder arthritis both in terms of clinical score (Figure 7A) and ankle swelling (Figure 7B); the control peptides $\mathrm{p} 277$ and V2E did not inhibit AA. The mean maximum AA score was $13.7 \pm 0.3$ in the control-treated rats compared with $7.3 \pm 0.7$ in the FP-treated rats $(P<0.05$ for the FP group compared with the control groups). These results correlated with inhibition of leg swelling: average leg swelling was $4.23 \pm 0.16 \mathrm{~mm}$ in control AA rats, and average leg swelling of FP-treated AA rats was significantly reduced to $2.24 \pm 0.18 \mathrm{~mm}(P<0.05)$. Treatment with the V2E mutant led to some reduction in average leg swelling $(3.33 \pm 0.21 \mathrm{~mm})$, but this reduction was significantly lower $(P<0.05)$ than that observed in FP-treated rats.

The activity of $\mathrm{T}$ cells that mediate AA can also be detected in vivo by studying the delayed-type hypersensitivity (DTH) response to PPD (30). We studied the DTH response to PPD 16 days after AA induction in rats treated with peptide FP, V2E, or p277. Figure 7C shows that the administration of FP led to a $35 \%$ reduction in the DTH response to PPD while the inhibition caused by treatment with the V2E or the p277 peptides was $25 \%$ and $10 \%$, respectively ( $P<0.05$ for the FP group compared with the control groups).

The T cells driving AA manifest a Th1 phenotype; they secrete relatively large amounts of IFN- $\gamma$ upon activation with Mt antigens such as HSP71 or Mt176-190 (20,21). In contrast, the control of AA by various treatments is usually accompanied by a decreased Th1 response $(20,21,31)$. Figure 7D shows that lymph node cells from FP-treated rats manifested a reduced secretion of IFN- $\gamma$ upon stimulation with mycobacterial antigens HSP71 or Mt176-190, compared with rats treated with control peptide $\mathrm{p} 277(P<0.05$ for the FP group compared to the control groups). Treatment with V2E affected IFN- $\gamma$ secretion only mildly (Figure 7D).

We also tested whether FP itself is immunogenic. Lymph node cells from FP-treated rats were isolated and incubated with FP in vitro. The cells produced no IFN- $\gamma$ when stimulated with FP (Figure 7E). However, lymph node cells from V2E-treated rats produced high amounts of IFN- $\gamma$ when stimulated with V2E (Figure $7 \mathrm{E})$. Note that the difference between the peptides is 1 amino acid 
A

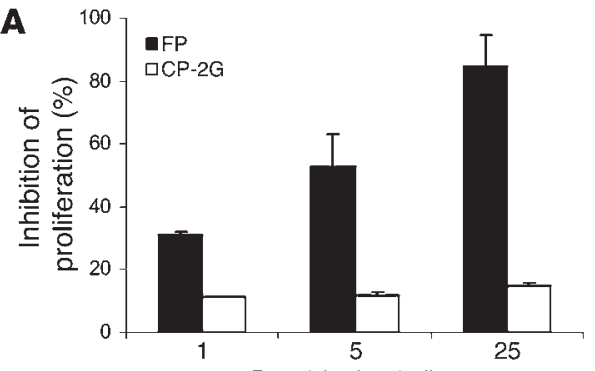

Peptide $(\mu \mathrm{g} / \mathrm{ml})$

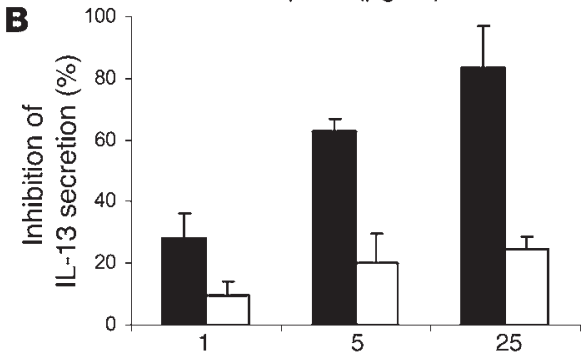

Peptide $(\mu \mathrm{g} / \mathrm{ml})$

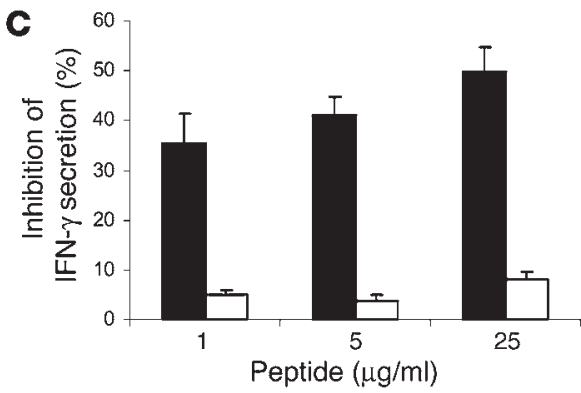

out of 33, yet this difference sufficed to block the immunogenicity of FP. This result is compatible with the conclusion that FP downregulates the $\mathrm{T}$ cell response to itself (Figure 7E).

Taken together, these results indicate that FP can interfere in vivo with the $\mathrm{T}$ cell activation induced by specific antigens. This interference led to milder AA (Figure 7, A and B), decreased DTH reactivity (Figure $7 \mathrm{C}$ ), and lower IFN- $\gamma$ secretion in response to $\mathrm{Mt}$ antigens (Figure 7D). Moreover, FP also downregulated its own immunogenicity (Figure 7E).

\section{Discussion}

In this work we report a new function for the HIV gp41 fusion domain: FP is able to suppress antigen-specific $\mathrm{T}$ cell activation. In vitro, FP inhibited the activation of primed lymph node cells or established human and rat $\mathrm{T}$ cell clones activated by specific antigens (Figures 3-5). In vivo, FP inhibited the activation of arthritogenic T cells and AA (Figure 7). These inhibitory effects were sequence specific, since the V2E mutant, differing from FP by only 1 out of 33 amino acids, was less active in vitro (Figures 3 and 5) and in vivo (Figure 7).

Our results indicate that FP targets the $\mathrm{T}$ cells rather than the APCs (Figure 5). FP has been reported to bind membrane domains in T cell-derived Jurkat cells; FP does not home to the cytoplasm (14). Using FRET, we found that FP inserts itself fewer than $50 \AA$ away from the TCR (Figure 2), an observation that was confirmed by the coimmunoprecipitation of FP with TCR molecules, suggesting that FP physically interacts with the TCR (Figure 6D and 6E).

\section{Figure 4}

FP inhibits the activation of human T cells. Human Ob1A12 cells were activated with their target peptide MBP85-99 and MGAR B cells, and proliferation (A), IL-13 secretion (B), and IFN- $\gamma$ secretion $(\mathbf{C})$ were assayed. The data are presented as mean inhibitions \pm SD $(n=3$ or more). The uninhibited T cell proliferative responses were $2440 \pm 190$ $\mathrm{cpm}$; the background proliferation in the absence of antigen was $85 \pm 12 \mathrm{cpm}$. IFN- $\gamma$ secretion was $964 \pm 149 \mathrm{pg} / \mathrm{ml}$, and IL-13 secretion was $4992 \pm 352 \mathrm{pg} / \mathrm{ml}$ for activated cells not incubated with FP or CP-2G. Two independent experiments gave similar results.

Note that FP did not inhibit T cell activation triggered by PMA/ionomycin or by mitogenic antibodies specific for the CD3 molecule (Figure 6, A and B). Both of these activators bypass the TCR (30); thus, we can conclude that FP most likely interacts with the TCR in a way that interferes with the TCR/CD3 crosstalk. This interference is likely to occur within the cell membrane since the V-to-E mutation present in the region of the $\mathrm{V} 2 \mathrm{E}$ peptide that inserts into the target membrane abrogated the coimmunoprecipitation of V2E with TCR molecules (Figure 6) and led to a decreased inhibitory activity (Figures 1, 3, 5, and 7). Interference with the TCR/CD3 interaction was postulated as the mechanism by which a synthetic 9-aa core peptide corresponding to the TCR- $\alpha$ transmembrane domain (CP) inhibits antigen-specific T cell activation $(23,24)$.

It has been reported previously that the 583-599 region in gp41 (Figure 1 and Table 1) harbors an immunosuppressive domain (ISU) $(32,33)$. ISU is capable of inhibiting T cell activation (32, 33). In contrast to FP, however, ISU can inhibit the T cell activation triggered by CD3-specific antibodies (32) or by PMA/ionomycin (34). Therefore, the molecular mechanisms of action of FP and ISU differ: ISU simultaneously targets protein kinase C activity (35) and the events related to $\mathrm{T}$ cell activation that occur within the cell membrane (36) while the FP only targets the latter, as demonstrated here. Hence, gp41 provides HIV with at least 2 different inhibitors of T cell activity: FP and ISU.

FP is usually hidden in the 3-dimensional structure of the gp41gp120 complex and is only briefly exposed during membrane fusion following changes in the structure of the gp41-gp120 complex (37). In the context of gp41, the brief exposure of FP to the target $\mathrm{T}$ cell membrane may suffice to activate not only its fusogenic activity but also its immunosuppressive function. However, it is still not clear whether gp41 has to be processed to release

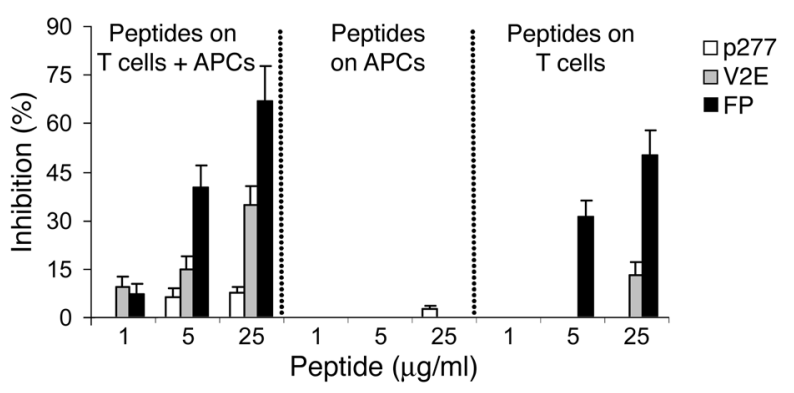

Figure 5

FP acts on T cells and not on APCs. A2b T cells or APCs were separately preincubated with $\mathrm{FP}, \mathrm{V} 2 \mathrm{E}$, or p277 for 2 hours, then washed. The treated T cells were mixed with untreated APCs, the treated APCs were mixed with untreated $A 2 b$ T cells, and the proliferation of the $A 2 b$ T cells upon stimulation with Mt176-190 was assayed. 
A
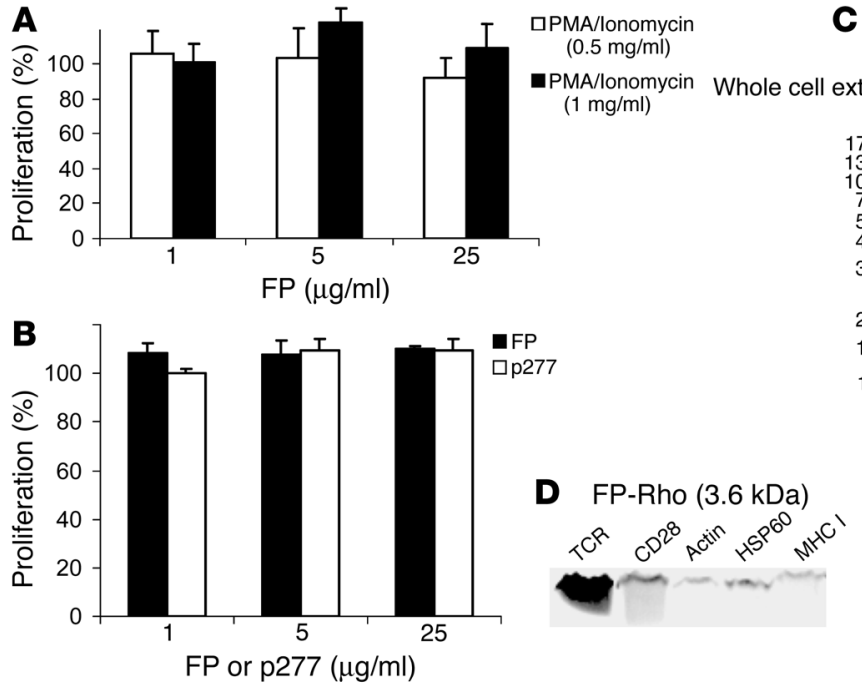

\section{C}

PMA/lonomycin Whole cell extract (1 mg/ml) (0.5 mg/ml)
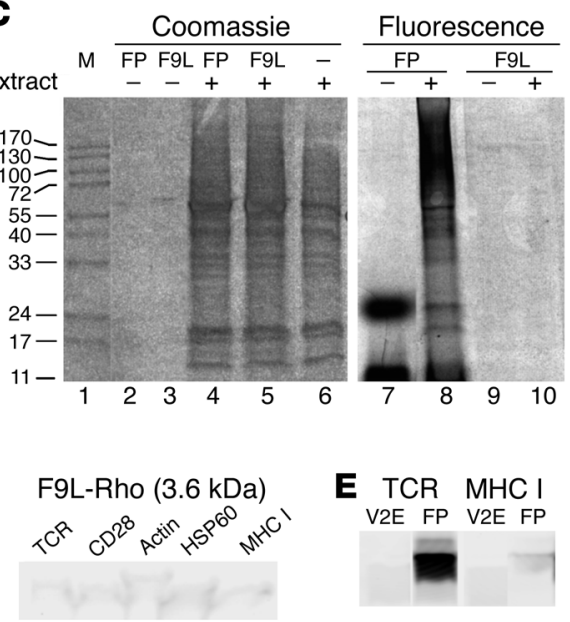

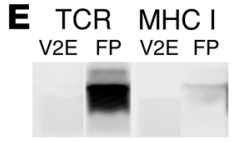

\section{Figure 6}

FP interacts with the TCR and does not inhibit T cell activation induced by PMA/ionomycin or antibodies to CD3. (A) A2b T cells were stimulated with PMA/ionomycin or (B) antibodies to CD3 $(1 \mu \mathrm{g} / \mathrm{ml})$ in the presence of FP or p277, and T cell proliferation was studied. (C) A2b T cells were incubated with FP-Rho or F9L-Rho overnight. Cells were cross-linked with DCC, and protein extracts were separated by SDS-PAGE and analyzed for rhodamine fluorescence. Total proteins were visualized by Coomassie brilliant blue. M, marker. (D) A2b T cells were incubated with FP-Rho or F9L-Rho for 1 hour, lysed, and immunoprecipitated with antibodies to TCR, CD28, actin, HSP60, or MHC I. Bound proteins were separated by SDS-PAGE and analyzed for the presence of the fluorescence-labeled peptides. (E) A2b T cells were incubated with FP-Rho or V2E-Rho for 1 hour, lysed, and immunoprecipitated with antibodies to TCR or MHC I. Bound proteins were separated by SDS-PAGE and analyzed for the presence of the fluorescence-labeled peptides.

active FP. In fact, it has been reported that whole gp41 and gp41 fragments can be released from infected cells after cytolysis (38). Moreover, sera of $\mathrm{HIV}^{+}$patients have been reported to contain a protein immunologically cross-reactive with gp41 that is capable of inhibiting monocyte chemotaxis (39).

The actual concentration of FP needed for immunosuppression may be lower in the context of the virus than the effective concentration that we observed for $\mathrm{FP}$ as a peptide $\left(\mathrm{IC}_{50} 280 \mathrm{nM}\right)$. The relatively high $\mathrm{IC}_{50}$ might reflect the self aggregation of the peptide (Figure 6C, lane 7), which leaves only a small fraction of FP free to insert itself into the $T$ cell membrane and exert its inhibitory effects. Indeed, this has also been observed for other peptides with immunosuppressive activity. For example, the immunosuppressive CP described by Manolios and coworkers has, in a similar experimental setup, an $\mathrm{IC}_{50}$ that is 100 -fold higher than that observed for FP (24).

It is conceivable that the immunomodulatory functions of FP are targeted preferentially to the HIV-specific immune response. Upon mucosal infection, DCs carry infective HIV virions attached to DC-SIGN molecules on their surface $(40,41)$ and in cellular compartments that protect the virions (42). At the lymph nodes, the DCs loaded with HIV can interact with HIV-specific T cells (43, 44), probably recruiting the HIV virions carried by the DCs to HIVspecific T cells (45). Once the virions reach the T cell membrane, FP might play 2 roles: mediating viral entry into the $\mathrm{T}$ cell (37) and, as indicated by our results, simultaneously inhibiting $\mathrm{T}$ cell activation. Since T cell activation can lead to the death of DCs (46), this suppressive effect of FP would increase DC survival and therefore enhance the chance of infection of other $\mathrm{T}$ cells interacting with the infected DCs. Obviously, the newly infected T cells must still undergo the minimal degree of activation needed to establish viral infection (47). Note that the inhibitory mechanism proposed here would preferentially contribute to the infection of HIV-specific T cells, as recently reported by Douek and colleagues (48), leaving $\mathrm{T}$ cells with other specificities relatively unharmed.

It is also conceivable that the suppressive activity of FP helps the virus cope with the selective pressure imposed by the immune system. One of the strategies used by HIV to escape the immune response is the continuous generation of new viral variants that require the host to continuously mount new adaptive immune responses $(4,49)$. However, the structural requirements needed to catalyze membrane fusion limit the genetic variability of FP; the structure of FP must remain compatible with effective HIV fusion to its target cells. The immunosuppressive activity of FP might contribute to conservation of the FP sequence and activity, protecting FP from the attack of antiviral T cell immunity. Several observations support this hypothesis. FP sequences are highly conserved regions of the HIV genome; the FP mutation rate is significantly lower than that of other genes in HIV (12). In addition, env products of the simian immunodeficiency virus that trigger a stronger impairment of $\mathrm{CD}^{+} \mathrm{T}$ cell responses show a higher fusogenic activity (50). Finally, the V2E peptide displays reduced fusogenic activity (9) and, in our experiments, reduced immunosuppressive function (Figures 3, 5, and 7); V2E, in contrast to FP, is immunogenic (Figure 7). Although it is still possible that the glutamic acid at position 2 of V2E is needed for MHC II binding (51), the increased immunogenicity of the V2E peptide might lie in its diminished immunosuppressive activity.

In conclusion, the present results reveal a novel aspect of the interaction of HIV with the host. In addition to its known role in membrane fusion, FP can collaborate in the downregulation of the immune response to HIV, supporting the conservation of 



viral sequences amid the selective pressure imposed by HIV-specific immunity. Note that FP administration inhibited in vitro the activation of human $\mathrm{T}$ cells involved in the pathology of multiple sclerosis (Figure 4) and in vivo, FP inhibited AA (Figure 7). Thus this immunosuppressive activity of FP might be exploited in the future for the design of new therapies for autoimmune disease (Figure 8). HIV has much to teach us about the regulation of the immune response.

\section{Methods}

Peptide synthesis. The peptides used in this work (Figure 1 and Table 1) were synthesized using a solid phase method on Rink amide resin $(0.15$ mequiv), as previously described $(9,52)$. The synthetic peptides were purified (greater than $98 \%$ homogeneity) by reverse-phase HPLC on a $\mathrm{C}_{4}$ column using a linear gradient of $20-60 \%$ acetonitrile in $0.05 \%$ trifluoroacetic acid (TFA) for 60 minutes. The peptides were subjected to aa analysis and mass spectrometry to confirm their composition. Unless stated otherwise, stock solutions of concentrated peptides were maintained in DMSO to avoid aggregation of the peptides prior to use. The final concentration of DMSO in each experiment $(5 \% \mathrm{vol} / \mathrm{vol})$ had no effect on the system under investigation. Table 1 provides the designations and sequences of the peptides used in this study.

Fluorescent labeling of peptides. The resin-bound peptides were treated with NBD-F or 5-carboxytetramethylrhodamine succinimidyl ester (rhodamine$\mathrm{SE}$ ). The NBD-F and rhodamine-SE fluorescent probes were purchased from Invitrogen Corp. The reaction with NBD-F took place in dimethylformamide (DMF), and the reaction with rhodamine in DMF containing $2 \%$

\section{Figure 7}

FP inhibits AA in rats. AA was induced by immunization to Mt in oil mixed with FP, V2E, p277, or PBS. (A) Arthritis was scored every 2 or 3 days starting at day 10; SEM was less than $10 \%$. The AA scores of the FP-treated group were significantly less than those of the control groups $(P<0.03)$. (B) DTH response to PPD was measured at day 16. (C) Leg swelling was measured at day 26. (D) IFN- $\gamma$ secretion was measured at day 26 upon stimulation of lymph node cells with HSP71 or Mt176-190. (E) IFN- $\gamma$ secretion from lymph node cells of PBS-, FP-, and V2E-treated rats was measured at day 26 upon stimulation with PBS, FP, and V2E, respectively. Lymph node cells from FP-treated rats showed no activation by FP.

diisopropylethylamine as described previously (26). The fluorescent probes were used in excess of 2 equivalents, leading to the formation of resin-bound $\mathrm{N}$ terminal NBD or rhodamine peptides. After 1 hour, the resins were washed thoroughly with DMF and then with methylene chloride. The resins were dried under nitrogen flow and then cleaved for 3 hours with 95\% TFA, 2.5\% $\mathrm{H}_{2} \mathrm{O}$, and $2.5 \%$ triethylsilane. The fluorescence-labeled peptides were purified by reverse-phase HPLC on a $\mathrm{C}_{4}$ Bio-Rad semipreparative column $(250 \times 10 \mathrm{~mm}$; pore size, $300 \AA$; particle size, $5 \mu \mathrm{m}$; Bio-Rad Laboratories) using a gradient of $20-60 \%$ of acetonitrile and water (both containing $0.05 \% \mathrm{TFA}$ ) for 60 minutes. The purified peptides were shown to be homogeneous (greater than $98 \%$ ) by analytical reverse-phase HPLC.

Cell lines, antigens, and adjuvants. The $\mathrm{CD}^{+}$rat $\mathrm{T}$ cell clone A2b (27) reacts with the 180-188 epitope of the mycobacterial HSP65. This epitope is contained in the Mt176-190 peptide and in the PPD antigen preparation used in our studies (53). A2b T cells were grown using APCs and the Mt176-190 peptide as described (27).

The $\mathrm{CD}^{+}$human Ob1A12 $\mathrm{T}$ cell clone was isolated from a patient with multiple sclerosis (22); it reacts with MBP85-99. Ob1A12 T cells were grown as previously described (54).

We purchased Difco Mt strain H37Ra and incomplete Freund's adjuvant (IFA) (BD Diagnostics). PPD was provided by the Statens Serum Institut. Purified recombinant HSP71 was generously provided by Ruurd van der Zee (Institute of Infectious Diseases and Immunology, Utrecht University, Utrecht, The Netherlands). PMA and ionomycin were purchased from Sigma-Aldrich.

Colocalization of peptides with membrane molecules. A2b cells that had been activated for 72 hours with PPD and APCs were fixed with $4 \%$ paraformaldehyde for 15 minutes on ice and washed with PBS. In control experiments, resting A2b cells (8-10 days after a previous activation by APC incubated with antigen) were used. A2b cells activated with PPD were also investigated without fixation with $4 \%$ paraformaldehyde. The cells were then treated with $2 \%$ BSA in PBS at room temperature to block unspecific binding. After 30 minutes, the cells were divided into aliquots containing 50,000 cells per $100 \mu \mathrm{l}$ and either anti-TCR-FITC or anti-CD4-FITC was added (1:100) and incubated for 2 hours. The rhodamine-labeled FP-Rho and V2E-Rho peptides were added during the last 5 minutes of incubation at a final concentration of $0.5-1 \mu \mathrm{M}$. The cells were then washed with PBS and deposited onto a glass slide. The labeled cell samples were observed under a fluorescence confocal microscope. FITC excitation was set at $488 \mathrm{~nm}$, with the laser set at $20 \%$ power to minimize 


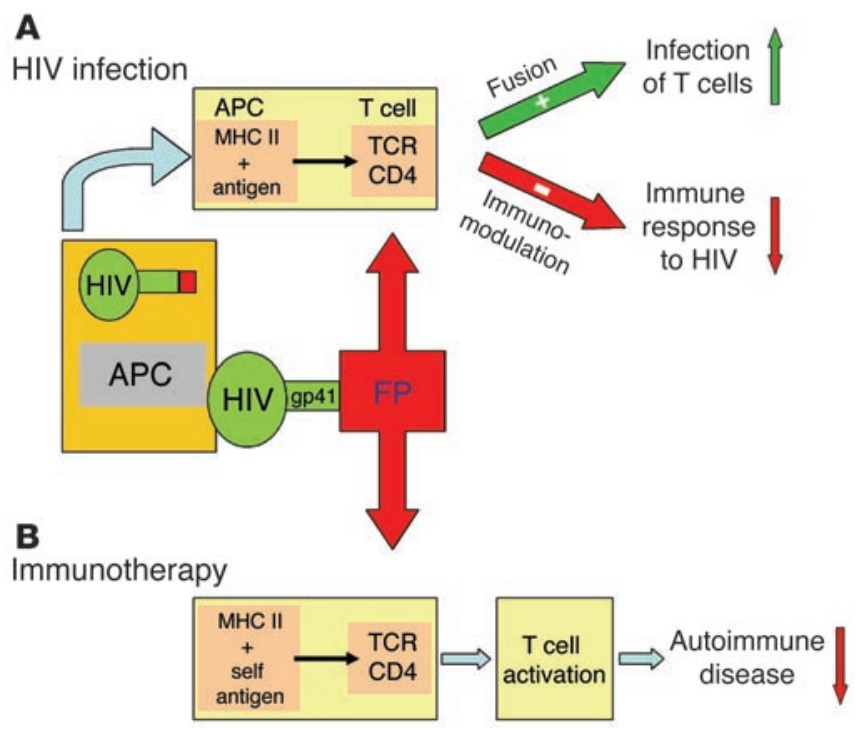

Figure 8

The hypotheses are that FP serves 2 functions in HIV infection and provides a new tool for immunotherapy. (A) HIV infection. Schematic illustration of 2 effects of FP on HIV infection. A DC infected with HIV, acting as an APC, presents processed HIV antigens via MHC class II to a $T$ cell. Insertion of FP into the T cell membrane facilitates fusion and infection while it downregulates specific T cell immunity to HIV epitopes. (B) Immunotherapy. Extension of the downregulation of AA mediated by FP, shown in this paper, to suggest that FP might serve as a new immunotherapeutic agent.

bleaching of the fluorophore. Fluorescence was recorded from 505-525 $\mathrm{nm}$. Rhodamine excitation was set at $543 \mathrm{~nm}$, with the laser set at $5 \%$ power. Fluorescence data were collected above $560 \mathrm{~nm}$.

FRET between FITC (donor label) and rhodamine (acceptor label) was detected by the increase in FITC fluorescence in a spot where the rhodamine probe was bleached. Bleaching was achieved by point excitation at $543 \mathrm{~nm}$ for 6 seconds with the laser set to $100 \%$. To verify that the increase in FITC fluorescence was not due to autofluorescence, we bleached first using the $488 \mathrm{~nm}$ laser and only afterward at $543 \mathrm{~nm}$. No signal was observed in either $505-525 \mathrm{~nm}$ or above $560 \mathrm{~nm}$, eliminating the possibility of autofluorescence.

$T$ cell activation and proliferation. $\mathrm{T}$ cell proliferation assays were performed using either rat lymph node cells, the rat A2b T cell clone (reactive with the Mt176-190 peptide), or the human Ob1A12 T cell clone (reactive with the MBP85-99 peptide).

Rat lymph node cells were prepared from popliteal and inguinal lymph nodes removed 26 days after the injection of Mt into IFA, when strong T cell responses to PPD and Mt176-190 were detectable (21). Lymph node cells were cultured at a concentration of $2 \times 10^{5}$ cells per well; $5 \times 10^{4} \mathrm{~A} 2 \mathrm{~b}$ T cells were stimulated with their target peptide Mt176-190 in the presence of $5 \times 10^{5}$ irradiated thymic APCs per well, prepared as previously described (30). The cells were plated in quadruplicates in 200- $\mu 1$ round-bottom microtiter wells (Corning Life Sciences) with or without PPD antigen or Mt176-190 peptide in the presence of various concentrations of the peptides under study. For some experiments, A2b cells were activated with immobilized anti-CD3 antibodies (55) or PMA/ ionomycin as described (23).

Ob1A12 T cells were stimulated using irradiated APCs fed with the target peptide MBP85-99 as described by Mycko and coworkers (54); the APCs were $B$ cells from the homozygous B cell line MGAR (54). T cell proliferation assays were set up in triplicate in 96-well U-bottom plates with $5 \times 10^{4} \mathrm{Ob} 1 \mathrm{~A} 12 \mathrm{~T}$ cells per well and $1 \times 10^{4}$ antigen-fed MGAR cells per well, as described (54).

Cultures were incubated for 72 hours at $37^{\circ} \mathrm{C}$ in a humidified atmosphere of $7.5 \% \mathrm{CO}_{2}$. T cell responses were detected by the incorporation of [methyl ${ }^{3} \mathrm{H}$ ]-thymidine ( $1 \mu \mathrm{Ci} /$ well; Amersham Biosciences), added during the last 18 hours of incubation. The results of $\mathrm{T}$ cell proliferation experiments are shown as the percentage of inhibition of the $\mathrm{T}$ cell proliferation triggered by the antigen in the absence of HIV or control peptides. In colocalization studies, A2b T cells were used after 72 hours of incubation with APCs and antigen. Resting A2b cells were also used for colocalization studies 8-10 days after a previous activation by APCs incubated with antigen (PPD or Mt176-190) as described above.

Cytokine assays. Rat IL-10 and IFN- $\gamma$ were quantified in supernatants collected after 72 hours of stimulation using the OPTEIA kit (BD Biosciences - Pharmingen) for ELISA as described (21). Human IFN- $\gamma$ and IL-13 were quantified in supernatants collected after 48 hours of stimulation by ELISA as described (56), using capture and detection antibody pairs and cytokine standards purchased from Endogen Inc. for IFN- $\gamma$ and from BD Biosciences - Pharmingen for IL-13. Avidin-peroxidase conjugate (Sigma-Aldrich) and TMB peroxidase substrate (KPL) were used to develop the assay.

Cytokine levels are expressed as percentages of cytokine inhibition relative to cytokine levels when no peptide is present. Otherwise, the levels of cytokine secretion are shown as $\mathrm{pg} / \mathrm{ml}$ in culture supernatants. Cytokine amounts were calculated based on calibration curves constructed using recombinant cytokines as standards.

Cross-linking of fluorescence-labeled peptides to $T$ cell membrane proteins. $\mathrm{A} 2 \mathrm{~b} \mathrm{~T}$ cells $\left(2 \times 10^{6}\right)$ were cultured overnight at $37^{\circ} \mathrm{C}$ in the presence of FP or F9L $(25 \mu \mathrm{g} / \mathrm{ml})$, or medium alone. The cells were then incubated with DCC (25) $(50 \mu \mathrm{g} / \mathrm{ml})$ for 1 hour at $37^{\circ} \mathrm{C}$ and lysed for 15 minutes on ice in $0.1-\mathrm{ml} \mathrm{lysis}$ buffer containing $10-\mathrm{mM}$ Tris, $137-\mathrm{mM} \mathrm{NaCl}, 10 \%$ glycerol, $1 \%$ digitonin, 2-mM EDTA, 0.4-mM PMSF, 4- $\mu \mathrm{g} / \mathrm{ml}$ leupeptin, and $4-\mu \mathrm{g} / \mathrm{ml}$ aprotinin (57) (Sigma-Aldrich). Insoluble material was removed by centrifugation at $10,000 \mathrm{~g}$ for 10 minutes at $4^{\circ} \mathrm{C}$. The proteins were resolved by PAGE-SDS, and total proteins were stained with Simply Blue SafeStain (Invitrogen Corp.) while proteins bound to fluorescence-labeled peptide were detected by the Typhoon 9400 variable mode imager (Amersham Biosciences). Excitation was set at $532 \mathrm{~nm}$, and fluorescence emission was collected by a TAMRA filter $(580 \mathrm{~nm} \pm 30 \mathrm{~nm})$ at $100 \mu \mathrm{m}$ resolution and 500 volts.

Immunoprecipitation of fluorescence-labeled peptides with $T$ cell proteins. Activated A2b T cells $\left(2 \times 10^{6}\right)$ were cultured for 1 hour at $37^{\circ} \mathrm{C}$ in the presence of FP or F9L $(25 \mu \mathrm{g} / \mathrm{ml})$ or medium alone and lysed for 15 minutes on ice in $0.1-\mathrm{ml}$ lysis buffer (57). Insoluble material was removed by centrifugation at $10,000 \mathrm{~g}$ for 10 minutes at $4^{\circ} \mathrm{C}$. The lysate was then incubated overnight with Protein A-plus Agarose beads (Santa Cruz Biotechnology Inc.) bound to antibodies to the rat TCR, CD28, actin, HSP60, or MHC class I. The antibodies reactive against the rat TCR (clone R73) or HSP60 (clone LK1) were purified from the respective hybridomas at our lab; antibodies to rat $\mathrm{CD} 28$, actin, and the rat $\mathrm{MHC}$ class I were purchased from Serotec. After an overnight incubation at $4{ }^{\circ} \mathrm{C}$, the beads were washed with lysis buffer and boiled for 10 minutes; the protein supernatant was then run in a $16 \%$ SDS-PAGE. The presence of coimmunoprecipitated peptide was detected by the Typhoon 9400 variable mode imager.

Animals. Three-month-old female Lewis rats were used in our experiments, raised and maintained under pathogen-free conditions in the Animal Breeding Center of the Weizmann Institute of Science. All animal experiments were approved by the Institutional Animal Care and Use Committee of the Weizmann Institute of Science.

Induction and assessment of $A A$. To test the effect of FP on $\mathrm{T}$ cell activation in vivo, we used AA as a model system. AA was induced in groups of 6 rats by injecting $50 \mu \mathrm{l}$ of Mt suspended in IFA $(0.5 \mathrm{mg} / \mathrm{ml})$ at the base of the 
tail. At the time of AA induction, each rat also received $100 \mu \mathrm{g}$ of FP or control peptide, or PBS dissolved in $50 \mu \mathrm{l}$ of IFA and mixed with Mt/IFA used to induce AA. The day of AA induction was designated as day 0 . Disease severity was assessed by direct observation of all 4 limbs in each animal. A relative score between 0 and 4 was assigned to each limb, based on the degree of joint inflammation, redness, and deformity; thus the maximum possible score for an individual animal was 16 (21). The mean AA score $( \pm$ SEM) is shown for each experimental group (Figure 7A). Arthritis was also quantified by measuring hind-limb diameter with a caliper. Measurements were taken on the day of the induction of AA and 26 days later (at the peak of AA); the results are presented as the mean \pm SEM of the difference between the 2 values for all the animals in each group. The person who scored the disease was blinded to the identity of the groups.

$D T H$. Twenty $\mu$ l of PPD $(0.5 \mathrm{mg} / \mathrm{ml}$ in PBS $)$ were injected intradermally into the pinna of the right ear on day 16 after AA induction in groups of 6 rats; $20 \mu \mathrm{l}$ of sterile PBS were injected into the pinna of the left ear as control. The thickness of the ear was measured 48 hours later using a vernier caliper and expressed as the difference between the right and the left ear.

Statistical significance. The InStat 2.01 program (GraphPad Software) was used for statistical analysis. Two-tailed Student's $t$ test and the Mann-Whitney $U$ test were conducted to assay significant differences between the different experimental groups. $P$ values less than 0.05 were considered significant.

\section{Acknowledgments}

We thank Vladimir Kiss for his help with the confocal microscopy, Batya Zarmi for her help with peptide purification, David Hafler for providing the Ob1A12 and MGAR cells, and Alex Kostianovsky for his help with the experiments on human T cells. We also thank Sergio G. Peisajovich for his helpful suggestions. Y. Shai holds the Harold S. and Harriet B. Brady Professorial Chair in Cancer Research, and I.R. Cohen is the Mauerberger Professor of Immunology at the Weizmann Institute of Science. This study was supported by the Minerva Foundation with funding from the Federal German Ministry for Education and Research.

Received for publication November 22, 2004, and accepted in revised form May 17, 2005.

Address correspondence to: Yechiel Shai, Department of Biological Chemistry, Weizmann Institute of Science, Rehovot 76100, Israel. Phone: 972-8-9342711; Fax: 972-8-9344112; E-mail: Yechiel.Shai@ weizmann.ac.il.

Francisco J. Quintana and Doron Gerber contributed equally to this work.
1. Douek, D.C., Picker, L.J., and Koup, R.A. 2003. T cell dynamics in HIV-1 infection. Annu. Rev. Immunol. 21:265-304.

2. Letvin, N.L., and Walker, B.D. 2003. Immunopathogenesis and immunotherapy in AIDS virus infections. Nat. Med. 9:861-866.

3. Stevenson, M. 2003. HIV-1 pathogenesis. Nat. Med. 9:853-860.

4. Johnson, W.E., and Desrosiers, R.C. 2002. Viral persistance: HIV's strategies of immune system evasion [review]. Annu. Rev. Med. 53:499-518.

5. Grossman, Z., Meier-Schellersheim, M., Sousa, A.E., Victorino, R.M., and Paul, W.E. 2002. CD4+ T-cell depletion in HIV infection: are we closer to understanding the cause? Nat. Med. 8:319-323.

6. Norris, P.J., and Rosenberg, E.S. 2001. Cellular immune response to human immunodeficiency virus. AIDS. 15:16-21.

7. Altfeld, M., and Rosenberg, E.S. 2000. The role of CD4(+) T helper cells in the cytotoxic T lymphocyte response to HIV-1. Curr. Opin. Immunol. 12:375-380.

8. Wyatt, R., and Sodroski, J. 1998. The HIV-1 envelope glycoproteins: fusogens, antigens, and immunogens [review]. Science. 280:1884-1888.

9. Kliger, Y., et al. 1997. Fusion peptides derived from the HIV type 1 glycoprotein 41 associate within phospholipid membranes and inhibit cell-cell fusion. Structure-function study. J. Biol. Chem. 272:13496-13505.

10. Peisajovich, S.G., and Shai, Y. 2003. Viral fusion proteins: multiple regions contribute to membrane fusion [review]. Biochim. Biophys. Acta. 1614:122-129.

11. Suarez, T., Nir, S., Goni, F.M., Saez-Cirion, A., and Nieva, J.L. 2000. The pre-transmembrane region of the human immunodeficiency virus type-1 glycoprotein: a novel fusogenic sequence. FEBS Lett. 477:145-149.

12. Chan, D.C., Fass, D., Berger, J.M., and Kim, P.S. 1997. Core structure of gp41 from the HIV envelope glycoprotein. Cell. 89:263-273.

13. Lawless, M.K., et al. 1996. HIV-1 membrane fusion mechanism: structural studies of the interactions between biologically-active peptides from gp41. Biochemistry. 35:13697-13708.

14. Cladera, J., Martin, I., and O'Shea, P. 2001. The fusion domain of HIV gp41 interacts specifically with heparan sulfate on the T-lymphocyte cell surface. $E M B O J$. 20:19-26.

15. Gerber, D., et al. 2004. Inhibition of HIV-1 glycoprotein-mediated cell fusion by a D,L-amino acid-containing fusion peptide: possible recognition of the fusion complex. J. Biol. Chem. 279:48224-48230.

16. Davis, D.M., and Dustin, M.L. 2004. What is the importance of the immunological synapse [review]? Trends Immunol. 25:323-327.

17. Huppa, J.B., Gleimer, M., Sumen, C., and Davis, M.M. 2003. Continuous T cell receptor signaling required for synapse maintenance and full effector potential. Nat. Immunol. 4:749-755.

18. Huppa, J.B., and Davis, M.M. 2003. T-cell-antigen recognition and the immunological synapse. Nat. Rev. Immunol. 3:973-983.

19. Heidecker, M., Yan-Marriott, Y., and Marriott, G. 1995. Proximity relationships and structural dynamics of the phalloidin binding site of actin filaments in solution and on single actin filaments on heavy meromyosin. Biochemistry. 34:11017-11025.

20. Quintana, F.J., Carmi, P., Mor, F., and Cohen, I.R. 2003. DNA fragments of the human $60-\mathrm{kDa}$ heat shock protein (HSP60) vaccinate against adjuvant arthritis: identification of a regulatory HSP60 peptide. J. Immunol. 171:3533-3541.

21. Quintana, F.J., Carmi, P., Mor, F., and Cohen, I.R. 2002. Inhibition of adjuvant arthritis by a DNA vaccine encoding human heat shock protein 60 . J. Immunol. 169:3422-3428.

22. Ota, K., et al. 1990. T-cell recognition of an immunodominant myelin basic protein epitope in multiple sclerosis. Nature. 346:183-187.

23. Wang, X.M., et al. 2002. T-cell antigen receptor peptides inhibit signal transduction within the membrane bilayer. Clin. Immunol. 105:199-207.

24. Manolios, N., et al. 1997. T-cell antigen receptor transmembrane peptides modulate $\mathrm{T}$-cell function and T cell-mediated disease. Nat. Med. 3:84-88.

25. Ballmer-Hofer, K., Schlup, V., Burn, P., and Burger, M.M. 1982. Isolation of in situ crosslinked ligandreceptor complexes using an anticrosslinker specific antibody. Anal. Biochem. 126:246-250.

26. Gerber, D., and Shai, Y. 2000. Insertion and organization within membranes of the delta-endotoxin pore-forming domain, helix 4-loop-helix 5, and inhibition of its activity by a mutant helix 4 peptide. J. Biol. Chem. 275:23602-23607.
27. Holoshitz, J., Matitiau, A., and Cohen, I.R. 1984. Arthritis induced in rats by cloned $\mathrm{T}$ lymphocytes responsive to mycobacteria but not to collagen type II. J. Clin. Invest. 73:211-215.

28. Holoshitz, J., Naparstek, Y., Ben-Nun, A., and Cohen, I.R. 1983. Lines of T lymphocytes induce or vaccinate against autoimmune arthritis. Science. 219:56-58.

29. Anderton, S.M., van der Zee, R., Noordzij, A., and van Eden, W. 1994. Differential mycobacterial 65-kDa heat shock protein $\mathrm{T}$ cell epitope recognition after adjuvant arthritis-inducing or protective immunization protocols. J. Immunol. 152:3656-3664.

30. van Eden, W., et al. 1985. Arthritis induced by a T-lymphocyte clone that responds to Mycobacterium tuberculosis and to cartilage proteoglycans. Proc. Natl. Acad. Sci. U. S. A. 82:5117-5120.

31. Tanaka, S., et al. 1999. Activation of T cells recognizing an epitope of heat-shock protein 70 can protect against rat adjuvant arthritis. J. Immunol. 163:5560-5565.

32. Ruegg, C.L., Monell, C.R., and Strand, M. 1989. Inhibition of lymphoproliferation by a synthetic peptide with sequence identity to gp 41 of human immunodeficiency virus type 1. J. Virol. 63:3257-3260.

33. Ruegg, C.L., Monell, C.R., and Strand, M. 1989. Identification, using synthetic peptides, of the minimum amino acid sequence from the retroviral transmembrane protein $\mathrm{p} 15 \mathrm{E}$ required for inhibition of lymphoproliferation and its similarity to gp21 of human T-lymphotropic virus types I and II. J. Virol. 63:3250-3256.

34. Ruegg, C.L., and Strand, M. 1991. A synthetic peptide with sequence identity to the transmembrane protein GP41 of HIV-1 inhibits distinct lymphocyte activation pathways dependent on protein kinase $\mathrm{C}$ and intracellular calcium influx. Cell Immunol. 137:1-13.

35. Ruegg, C.L., and Strand, M. 1990. Inhibition of protein kinase $\mathrm{C}$ and anti-CD3-induced Ca2+ influx in Jurkat $\mathrm{T}$ cells by a synthetic peptide with sequence identity to HIV-1 gp41. J. Immunol. 144:3928-3935.

36. Moreno, M.R., Pascual, R., and Villalain, J. 2004. Identification of membrane-active regions of the HIV-1 envelope glycoprotein gp41 using a 15-mer gp41peptide scan. Biochim. Biophys. Acta. 1661:97-105.

37. Peisajovich, S.G., and Shai, Y. 2002. New insights into the mechanism of virus-induced membrane 
fusion. Trends Biochem. Sci. 27:183-190

38. Parren, P.W., Burton, D.R., and Sattentau, Q.J. 1997. HIV-1 antibody-debris or virion? Nat. Med. 3:366-367.

39. Tas, M., Drexhage, H.A., and Goudsmit, J. 1988. A monocyte chemotaxis inhibiting factor in serum of HIV infected men shares epitopes with the HIV transmembrane protein gp41. Clin. Exp. Immunol. 71:13-18.

40. van Kooyk, Y., and Geijtenbeek, T.B. 2003. DCSIGN: escape mechanism for pathogens [review]. Nat. Rev. Immunol. 3:697-709.

41. Geijtenbeek, T.B., et al. 2000. DC-SIGN, a dendritic cell-specific HIV-1-binding protein that enhances trans-infection of T cells. Cell. 100:587-597.

42. Kwon, D.S., Gregorio, G., Bitton, N., Hendrickson, W.A., and Littman, D.R. 2002. DC-SIGN-mediated internalization of HIV is required for trans-enhancement of T cell infection. Immunity. 16:135-144.

43. Pope, M., et al. 1994. Conjugates of dendritic cells and memory $\mathrm{T}$ lymphocytes from skin facilitate productive infection with HIV-1. Cell. 78:389-398.

44. Sugaya, M., Lore, K., Koup, R.A., Douek, D.C., and Blauvelt, A. 2004. HIV-infected Langerhans cells preferentially transmit virus to proliferat- ing autologous CD4+ memory $\mathrm{T}$ cells located within Langerhans cell-T cell clusters. J. Immunol. 172:2219-2224.

45. McDonald, D., et al. 2003. Recruitment of HIV and its receptors to dendritic cell-T cell junctions. Science. 300:1295-1297.

46. Matsue, H., et al. 1999. Dendritic cells undergo rapid apoptosis in vitro during antigen-specific interaction with CD4+ T cells. J. Immunol. 162:5287-5298.

47. Woods, T.C., Roberts, B.D., Butera, S.T., and Folks, T.M. 1997. Loss of inducible virus in CD45RA naive cells after human immunodeficiency virus-1 entry accounts for preferential viral replication in CD45R memory cells. Blood. 89:1635-1641.

48. Douek, D.C., et al. 2002. HIV preferentially infects HIV-specific CD4+ T cells. Nature. 417:95-98.

49. Desrosiers, R.C. 1999. Strategies used by human immunodeficiency virus that allow persistent viral replication. Nat. Med. 5:723-725.

50. Karlsson, G.B., et al. 1998. The envelope glycoprotein ectodomains determine the efficiency of CD4+ $\mathrm{T}$ lymphocyte depletion in simian-human immunodeficiency virus-infected macaques. J. Exp. Med. 188:1159-1171.
51. Reizis, B., et al. 1996. The peptide binding specificity of the MHC class II I-A molecule of the Lewis rat, RT1.BI. Int. Immunol. 8:1825-1832.

52. Merrifield, R.B., Vizioli, L.D., and Boman, H.G. 1982. Synthesis of the antibacterial peptide cecropin A (1-33). Biochemistry. 21:5020-5031.

53. van Eden, W., et al. 1988. Cloning of the mycobacterial epitope recognized by $\mathrm{T}$ lymphocytes in adjuvant arthritis. Nature. 331:171-173.

54. Mycko, M.P., et al. 2004. Cross-reactive TCR responses to self antigens presented by different MHC class II molecules. J. Immunol. 173:1689-1698.

55. Kaleeba, J.A., Offner, H., Vandenbark, A.A., Lublinski, A., and Weinberg, A.D. 1998. The OX-40 receptor provides a potent co-stimulatory signal capable of inducing encephalitogenicity in myelin-specific CD4+ T cells. Int. Immunol. 10:453-461.

56. Viglietta, V., Kent, S.C., Orban, T., and Hafler, D.A. 2002. GAD65-reactive T cells are activated in patients with autoimmune type 1 a diabetes. J. Clin. Invest. 109:895-903. doi:10.1172/JCI200214114.

57. Adachi, T., et al. 1996. The specificity of association of the IgD molecule with the accessory proteins BAP31/BAP29 lies in the IgD transmembrane sequence. EMBOJ. 15:1534-1541. 